EPJ Web of Conferences 59, 17004 (2013)

DOI: $10.1051 /$ epjconf/20135917004

(C) Owned by the authors, published by EDP Sciences, 2013

\title{
Effects of laser temporal profile on fast electron characteristics
}

\author{
M. Hata ${ }^{1, a}$, H. Sakagami ${ }^{2}$, T. Johzaki ${ }^{3}$ and H. Nagatomo ${ }^{4}$ \\ 1 Department of Physics, Nagoya University, Nagoya Aichi 464-8602, Japan \\ 2 Fundamental Physics Simulation Research Division, National Institute for Fusion Science, \\ Toki Gifu 509-5292, Japan \\ 3 Institute of Laser Technology, Suita, Osaka 565-0871, Japan \\ 4 Institute of Laser Engineering, Osaka University, Suita, Osaka 565-0871, Japan
}

\begin{abstract}
D PIC simulations for fast ignition with a planar target and an ultrahigh intensity laser have been performed to investigate effects of laser temporal profile on fast electron characteristics. Six different laser profiles, which are combination of two pulse shapes, namely Flattop and Gaussian shapes, and three different maximum intensities $1 \times 10^{20}, 5 \times 10^{19}$, and $2.5 \times 10^{19} \mathrm{~W} / \mathrm{cm}^{2}$, are studied under the condition of fixed laser energy. Time-integrated energy spectra, coupling efficiencies from the heating laser to fast electrons, and divergence angle of fast electrons are shown and discussed.
\end{abstract}

\section{INTRODUCTION}

In 1994, the concept of fast ignition was proposed [1]. Many studies have intensively been performed since this proposal [2, 3]. In Japan, the FIREX-I project [4] has been started for the sake of heating the core up to the temperature of $5 \mathrm{keV}$. Cone-guided targets are used in this project and the design optimization of the targets has been performed $[5,6]$. In contrast, the profile of the ultrahigh intensity laser that heats the core is not well optimized and its effects on fast electron generation are not clarified yet. Therefore, it is necessary for full optimization to investigate effects of laser temporal and spatial profiles. In this paper, we pay attention to the laser temporal profile and estimate its effects on fast electron characteristics.

\section{FAST ELECTRON CHARACTERISTICS}

We simulated laser-plasma interactions for normal incidence of an ultrahigh intensity laser onto a gold planar target with a two-dimensional PIC code, FISCOF2 [7]. A prepulse of the heating laser is intense enough (e.g., $10^{13} \mathrm{~W} / \mathrm{cm}^{2}$ ) to create a plasma because the intensity of the main pulse is extremely high (e.g., $10^{20} \mathrm{~W} / \mathrm{cm}^{2}$ ) even if the contrast ratio is $10^{7}$. As a result, the target is ionized before the main pulse reaches the target, and a preformed plasma (preplasma) is generated. Therefore, we assume the target is ionized and do not treat ionization process in simulations. Further, collision effects are neglected because we simulate only fast electron generation that occurs in relatively low-density plasma. In order to investigate effects of the laser temporal profile, we studied six different cases that are combination of two pulse shapes, namely Flattop and Gaussian shapes, and three different maximum intensities $1 \times 10^{20}$, $5 \times 10^{19}$, and $2.5 \times 10^{19} \mathrm{~W} / \mathrm{cm}^{2}$ as summarized in Table 1 . The laser wavelength is set to $1.06 \mu \mathrm{m}$.

\footnotetext{
${ }^{a}$ e-mail: hata.masayasu@nifs .ac.jp
}

This is an Open Access article distributed under the terms of the Creative Commons Attribution License 2.0, which permits unrestricted use, distribution, and reproduction in any medium, provided the original work is properly cited. 


\section{EPJ Web of Conferences}

Table 1. Laser maximum intensities, temporal profiles, and coupling efficiencies of laser to observed electrons with energies of all range, $0-0.2,0.2-2,2-7 \mathrm{MeV}$, and more than $7 \mathrm{MeV}$ in each laser profile cases.

\begin{tabular}{|l|l|l|c|c|c|c|c|}
\hline Case & $\begin{array}{l}\text { Maximum } \\
\text { intensity }\end{array}$ & $\begin{array}{l}\text { Temporal } \\
\text { profile }\end{array}$ & \multicolumn{5}{|c|}{ Coupling efficiencies of each electron energy range [\%] } \\
\hline & $I\left[\mathrm{~W} / \mathrm{cm}^{2}\right]$ & $\begin{array}{l}\tau_{\text {rise/fall }}, \\
\tau_{\text {flat }}[\mathrm{fs}]\end{array}$ & All range & $0-0.2 \mathrm{MeV}$ & $0.2-2 \mathrm{MeV}$ & $2-7 \mathrm{MeV}$ & $>7 \mathrm{MeV}$ \\
\hline FH & $1.0 \times 10^{20}$ & $4.7,90$ & 71.96 & 9.20 & 5.44 & 18.50 & 38.83 \\
\hline FM & $5.0 \times 10^{19}$ & $4.7,190$ & 78.78 & 11.8 & 10.24 & 26.91 & 29.88 \\
\hline FL & $2.5 \times 10^{19}$ & $4.7,390$ & 82.28 & 13.02 & 16.67 & 33.41 & 19.19 \\
\hline GH & $1.0 \times 10^{20}$ & 94,0 & 78.49 & 11.36 & 8.65 & 21.87 & 36.62 \\
\hline GM & $5.0 \times 10^{19}$ & 188,0 & 81.53 & 12.60 & 14.74 & 29.72 & 24.48 \\
\hline GL & $2.5 \times 10^{19}$ & 376,0 & 86.41 & 12.01 & 21.18 & 35.74 & 17.48 \\
\hline
\end{tabular}

Laser pulses were assumed to be uniform in y direction and pulse lengths are adjusted to preserve the total energy. The intensity in the case of Flattop pulses rose up with a Gaussian profile having half-width at half maximum (HWHM) of $4.7 \mathrm{fs}$, was kept constant with its corresponding maximum intensity during 90, 190, or $390 \mathrm{fs}$, and fell down with a Gaussian profile having HWHM of $4.7 \mathrm{fs}$. These three cases are called FH (Flattop of High-intensity), FM (Flattop of Medium-intensity), and FL (Flattop of Low-intensity). In the other three cases, the intensity profile is Gaussian with full-width at half maximum of 94, 188, or $376 \mathrm{fs}$ (corresponding maximum intensities of $1 \times 10^{20}, 5 \times 10^{19}$, and $2.5 \times 10^{19} \mathrm{~W} / \mathrm{cm}^{2}$ ) and they are called GH (Gaussian of High-intensity), GM (Gaussian of Mediumintensity), and GL (Gaussian of Low-intensity). The electron density profile is uniform in y direction, and that in $\mathrm{x}$ direction consists of exponential region with the scale length $4 \mu \mathrm{m}$ from 0.1 to $20 \mathrm{n}_{\mathrm{cr}}$ and flat region with the thickness of $20 \mu \mathrm{m}$ and the density of $20 \mathrm{n}_{\mathrm{cr}}$, where $\mathrm{n}_{\mathrm{cr}}$ means the critical density and the exponential region represents the preplasma. The charge state, mass number, and proton-to-electron mass ratio were set to 40,197, and 1836, respectively. The electron and ion initial temperatures were assumed to be 10 and $1 \mathrm{keV}$, respectively. Boundary conditions in the $\mathrm{x}$ direction are the reflection for particles and absorption for fields, and those in the y direction are periodic for both particles and fields. Generated fast electrons were observed in the plasma, $5 \mu \mathrm{m}$ behind the boundary between the preplasma and the flat profile plasma. To ignore the circulation of fast electrons, fast electrons were artificially cooled to the initial temperature in the rear part, from the rear edge of the plasma to $6 \mu \mathrm{m}$ inner side.

\subsection{Time-integrated energy spectra}

Figure 1 shows time-integrated energy spectra of fast electrons, where the integration time is $2000 \mathrm{fs}$. In the cases of flattop pulses, low-energy $(<7 \mathrm{MeV})$ electrons decrease and high-energy ( $>7 \mathrm{MeV}$ ) electrons increase as the maximum laser intensity becomes higher. High-energy electrons are more generated and the number of low-energy electrons decreases in the high-intensity case compared to the low-intensity case. The electron slope temperatures in the spectra are somewhat higher than those of Wilks's scaling [8] by reason of preplasma effects. According to Cai et al.'s research related to the preplasma effects on fast electron generation [9], the slope temperature becomes high because of stochastic heating [10] in the underdense plasma if the preplasma exists. In our simulations, the reflected laser intensity is approximately $20 \%$ of the incident laser intensity in all cases, therefore the amplitudes of two pulses exceed thresholds for stochastic motion and then stochastic heating occurs. As to the cases of Gaussian pulses in Figure 1, the trend of the spectra is the same as that of the flattop cases. When the cases of the same maximum intensities are compared, the generation of high-energy electrons is 


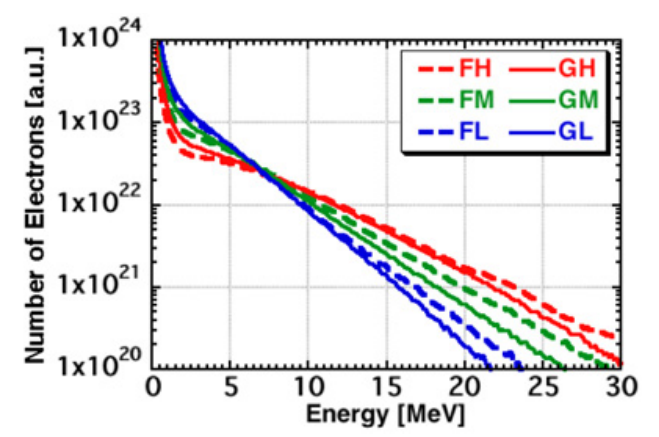

Figure 1. Time-integrated electron energy spectra. Integration time is $2000 \mathrm{fs}$.
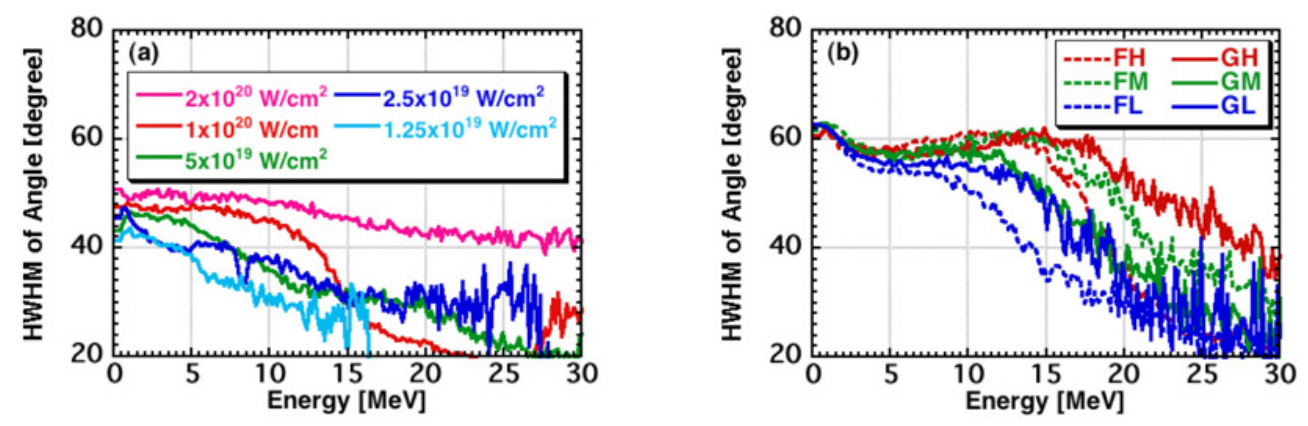

Figure 2. HWHMs as a function of electron energy on time-integrated electron mometum distributions in the cases of (a) flattop with same pulse duration of $100 \mathrm{fs}$ and five different maximum intensities of $2 \times 10^{20}, 1 \times 10^{20}$, $5 \times 10^{19}, 2.5 \times 10^{19}$, and $1.25 \times 10^{19} \mathrm{~W} / \mathrm{cm}^{2}$ and (b) simulations of previous subsection.

enhanced and that of low-energy electrons is weakened in the case of flattop compared to the Gaussian case because the laser intensity of the Gaussian case is equal or lower than that of the flattop case. Coupling efficiencies to observed electrons with energies of all range, $0-0.2,0.2-2,2-7 \mathrm{MeV}$, and more than $7 \mathrm{MeV}$ are summarized in Table 1 . The difference of $0.2-2 \mathrm{MeV}$ electrons in Table 1 is especially large compared to the others. The results are remarkable because these electrons efficiently heat the core. Therefore the spectrum of the GL case is the most suitable for fast ignition in those simulations since these electrons are much generated.

\subsection{Divergence angle of fast electrons}

To evaluate intensity dependence of divergence angle for electrons, we performed simulations with only flattop pulse shape and same pulse durations of $100 \mathrm{fs}$ but five different maximum intensities of $2 \times 10^{20}, 1 \times 10^{20}, 5 \times 10^{19}, 2.5 \times 10^{19}$, and $1.25 \times 10^{19} \mathrm{~W} / \mathrm{cm}^{2}$, where other conditions were the same as those of previous simulations. We calculate HWHMs of divergence angle from the timeintegrated electron momentum distributions over $200 \mathrm{fs}$. Figure 2(a) shows HWHMs as a function of the electron energy. The divergence angle tends to be larger as the intensity becomes higher. Its trend is similar to experimental and theoretical results of Reference [11]. It is noticed that HWHMs for electrons with $15-25 \mathrm{MeV}$ energy in the case of $1 \times 10^{20} \mathrm{~W} / \mathrm{cm}^{2}$ is the smallest in five cases, but its reason is still inexplicable. We also analyze results of previous simulations. Figure 2(b) shows HWHMs of divergence angle as a function of the electron energy calculated from the time-integrated electron momentum distributions over $2000 \mathrm{fs}$. In all cases, the HWHM becomes small with increasing electron 


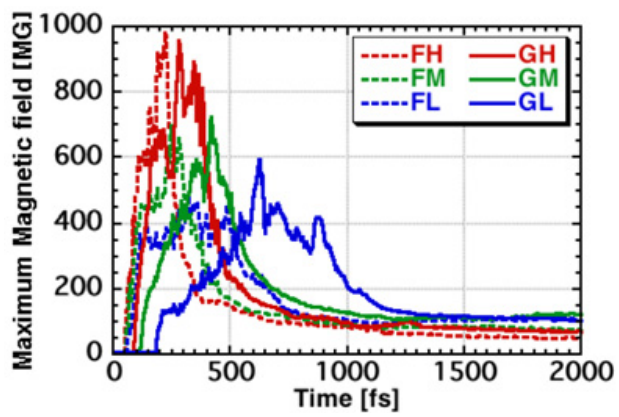

Figure 3. Time evolutions of the maximum magnetic field.

energy because high-energy electrons are hard to be scattered by the magnetic field. Figure 3 shows time evolutions of the maximum magnetic field. Large magnetic fields are observed and peak of the maximum magnetic field increases with the maximum laser intensity. It is considered that the divergence angle for electrons with same energy becomes larger with the laser intensity, because the generated electron beam is also more intense and it causes larger magnetic fields. In the comparison of all cases in Figure 2(b), divergence angles for low-energy electrons are similar. On the other hand, divergence angles for highenergy electrons in the case of FL are the smallest and those of the GH case are the largest. To clarify these relations between the divergence angle and laser temporal profile, more simulations and detailed analysis are needed.

\section{SUMMARY}

We simulated the cases of six different laser profiles under the condition of fixed laser energy, and investigated effects of laser temporal profile on fast electron characteristics. Results show that the generation of low-energy electrons are weakened and high-energy electrons are much generated as the laser intensity becomes high. In addition, divergence angles of low-energy electrons were similar in all cases because of the strong magnetic fields. In contrast, those of high-energy electrons were different. Consequently, the low-intensity Gaussian laser pulse is clearly the most suitable for fast ignition within the simulated parameters, because low-energy electrons that heat the core efficiently are much generated and their divergence angles are similar to those of other cases.

\section{References}

[1] M. Tabak, J. Hammer, M.E. Glinsky et al., Phys. Plasmas 1, 1626 (1994)

[2] M. Tabak, D. Clark, S. Hatchett et al., Phys. Plasmas 12, 057305 (2005)

[3] P. Norreys, R. Scott, K. Lancaster et al., Nucl. Fusion 49, 104023 (2009)

[4] H. Azechi and The FIREX Project., Plasma Phys. Control. Fusion 48, B267 (2006)

[5] H. Sakagmi, T. Johzaki, H. Nagatomo, K. Mima, Nucl. Fusion 49, 075026 (2009)

[6] T. Johzaki, H. Nagatomo, A. Sunahara et al., Nucl. Fusion 51, 073022 (2011)

[7] H. Sakagami and K. Mima, Proc. $2^{\text {nd }}$ Int. Conf. on Inertial Fusion Sciences and Applications (Kyoto, Japan, 9-14 September 2001), 380 (2002)

[8] S. C. Wilks, W. L. Kruer, M. Tabak, A. B. Langdon, Phys. Rev. Lett. 69, 1383 (1992)

[9] H. Cai, K. Mima, A. Sunahara et al., Phys. Plasmas 17, 023106 (2010)

[10] Z. M. Sheng, K. Mima, J. Zhang et al., Phys. Rev. E 69, 016407 (2004)

[11] J.S. Green, V.M. Ovchinnikov, R. G. Evans et al., Phys. Rev. Lett. 100, 015003 (2008) 